eISSN: 2659-6482

DOI: https://doi.org/10.14201/pmrt.23934

\title{
EL PROGRAMA DE MÚSICA DE LAS ESCUELAS DE NIÑAS DURANTE LA DICTADURA FRANQUISTA: UNA MIRADA A LOS CUESTIONARIOS NACIONALES DE 1953
}

\section{The Music's Program of the Girls School during the Franco Dictatorship: A look at the National Questionnaries of 1953}

\author{
Alba María LÓPEZ MELGAREJO \\ (ISEN Cartagena) \\ albamaria.lopez@um.es
}

RESUMEN: El proceso de inclusión de la enseñanza de la Educación Musical en la escuela en España se ha caracterizado por una lenta evolución. Con la ley de 1945 algunas áreas presentaron diferencias en razón de género, siendo la Música una de ellas. Así pues, este trabajo pretende volver la mirada al punto de partida de su creación a través de un análisis documental que profundiza en la enseñanza musical para las escuelas de niñas, tal como se recoge en los Cuestionarios Nacionales de 1953. Un predominio de las canciones de corro para el nivel más elemental de instrucción primaria junto a religiosas, himnos o tradicionales españolas son algunas de las inclusiones en el currículo del momento para las niñas. Si bien, la inclusión de contenidos mucho más elementales para ellas frente a la propuesta de contenidos para los niños refleja un menor interés por parte del Gobierno nacional hacia la formación de estas.

Palabras clave: Educación musical; currículo; franquismo; niñas.

ABSTRACT: The process of including the teaching of Music Education at school in Spain has been characterized by a slow evolution. With the 1945 law, some areas presented differences based on gender, with Music being one of them. Thus, this work aims to return the gaze to the starting point of its creation through a documentary analysis that delves into music 
teaching for girls' schools, as reflected in the 1953 National Questionnaires. A predominance of songs for the most elementary level of primary education together with religious, hymns or traditional Spanish are some of the inclusions in the current curriculum for girls. Although, the inclusion of much more elementary content for them compared to the proposal of content for children reflects a lower interest on the part of the national Government towards their formation.

Keywords: Music education; curriculum; Francoism; girls.

\section{INTRODUCCIÓN}

Finalizada la Guerra Civil española e instaurada la Dictadura franquista, la educación española sufrió un retroceso tras los avances alcanzados durante la II República. En 1945 se publicaba la Ley de Educación basada en la familia, el Estado y la Iglesia. Con el objetivo de controlar las enseñanzas impartidas en los centros públicos, se publicaron en 1953 los Cuestionarios Nacionales a modo de currículo para la Enseñanza Primaria, siendo meramente orientativos para las instituciones de gestión privada, que debían contar con «una conducta religiosa y moral intachable en la persona individual que dirija la Escuela u orientación del mismo carácter en la colectividad que la sostenga» (Ley de 17 de julio, 1945, p. 392). Dicho de otro modo, los centros de titularidad privada estaban en su mayoría en manos de la Iglesia católica y, por tanto, no era necesario ejercer un control sobre ellos a diferencia de los de gestión pública.

Dicho programa de estudios establece tanto las áreas escolares como los contenidos a impartir en las aulas. Si bien, son muchas las diferencias existentes para las asignaturas que se consideraban propias del plan de estudios que debían seguir los niños y aquellas materias destinadas al conocimiento por parte de las niñas, entre ellas, la Música.

Este trabajo tiene como objetivo ofrecer un análisis de los contenidos del área de Música presentes en los Cuestionarios Nacionales de 1953 para la enseñanza de las niñas. Para ello, se ha realizado un análisis documental de los aspectos musicales recogidos en el documento. Al mismo tiempo, se ha considerado preciso realizar en algún apartado alusión a la propuesta destinada a los niños, con el fin de ofrecer a su vez una perspectiva de las diferencias en razón de género que determinaron la enseñanza musical de la década de los cincuenta y principio de los sesenta en España en las escuelas de niñas. 
ALBA MARÍA LÓPEZ MELGAREJO

EL PROGRAMA DE MÚSICA DE LAS ESCUELAS DE NIÑAS DURANTE LA DICTADURA FRANQUISTA:

UNA MIRADA A LOS CUESTIONARIOS NACIONALES DE 1953

\section{LA ESCOLARIZACIÓN OBLIGATORIA Y LOS ANTECEDENTES AL CURRÍCULO DE MÚSICA}

La obligatoriedad de los planes de estudios no puede ser entendida sin comprender el proceso de escolarización obligatoria en España que se inicia con la Constitución de 1812. Los ideales y postulados defendidos por la Revolución francesa convirtieron a la Educación en un derecho de la ciudadanía (Lázaro, 1993). Junto a ellos, los principios promovidos por la Ilustración en España, así como las políticas acometidas por el gobierno de Carlos III durante el siglo XVIII (Domínguez, 1990), fueron claves para que la gestión de la Instrucción Primaria pasara a manos del Estado a lo largo del siglo XIX. Este proceso fue lento y en gran medida de carácter teórico, al dictar mucha diferencia entre lo que se legisló y la realidad educativa presente en la sociedad de la época. Así pues, el artículo 366 del título IX de la Constitución de 1812 establece para el territorio del Reino de España que «se establecerán escuelas de primeras letras, en las que se enseñará a los niños a leer, escribir y contar, y el catecismo de la religión católica, que comprenderá también una breve exposición de las obligaciones civiles» (1812, p. 46).

En este primer momento, el deber de educar a los niños recae en los padres y no en el Estado, quienes debían enseñar a sus hijos, ya fuera a través de la asistencia a la escuela o en casa, una serie de aprendizajes concretos. Este principio recogido en la Constitución dio luz verde para que durante la primera mitad del XIX se publicaran numerosos documentos que intentaron regular la Educación, siendo todos ellos los antecedentes legislativos de la Ley Moyano publicada en 1857 y vigente hasta 1970.

En este contexto surge el Informe Quintana en 1813 (Morales, 2008), que, a pesar de los postulados básicos a favor de una educación universal y uniforme, defendida en la Constitución de 1812, muestra una gran diferencia de la concepción de la educación en razón de género. Así pues, «la educación de la mujer se concebía de índole moral, doméstica y privada. [...] se consideraba que era suficiente con escuelas públicas, donde se enseñará (sic) a las niñas a leer y escribir, y a las adultas, las labores propias de su sexo [...]» (Araque, 2009: 14). Junto a este documento, se ha de destacar también la importancia e influencia que tuvieron el Reglamento General de Instrucción Pública de 1821 o el Plan del Duque de Rivas en 1838 en la elaboración de la Ley Moyano.

La promulgación de la Ley Moyano el 9 de septiembre de 1857 reconfiguró el sistema educativo. Sin lugar a dudas, fue el reconocimiento de la obligatoriedad y gratuidad de la enseñanza para los niños de entre 6 a 9 años su rasgo más significativo. La Instrucción Primaria se configuró en dos etapas siendo la primera de ellas elemental y obligatoria (6-9 años) y la segunda superior y optativa (9-12 años). Para garantizar la uniformidad de los contenidos a impartir en todo el territorio nacional «la enseñanza debía llevarse a cabo por medio de libros señalados por el gobierno» 
(Egido, 1995: 80). Dicha normativa también fue meramente teórica. Aun así, entre los contenidos a impartir en las escuelas figuraba la enseñanza de la lectura, la escritura, la doctrina cristiana, conocimientos básicos de geografía y la iniciación a la gramática y aritmética, «y si el maestro o maestra tenían la preparación adecuada y suficientes recursos, a ciertas nociones de industria, comercio y agricultura, sustituidas para las niñas por labores propias de su sexo» (Puelles, 1999: 421). Tristemente, la música no formó parte de estas primeras enseñanzas mínimas.

La situación socioeconómica y cultural en España durante la segunda mitad del XIX, la crisis económica, la sociedad rural, junto a las desigualdades sociales, justifican un lento proceso de la escolarización efectiva en España. Tres décadas después de la entrada en vigor de la Ley Moyano, el Código Civil de 1888 aún aludía a la tarea alfabetizadora como una responsabilidad de los padres o tutores. Como peculiaridad, se establece que la educación que estos debían ofrecer a sus hijos o a los menores que estuvieran bajo su tutela debía variar en razón del nivel adquisitivo de la familia. Esto supone una tendencia a la reproducción de las clases sociales.

Con la entrada en el siglo XX, la creación del Ministerio de Instrucción Pública (1900) fue decisiva para la consolidación de «ese proceso irreversible de la estatalización de la enseñanza» (Ávila, 2011: 440). El murciano Antonio García Alix inició una reforma del sistema educativo que, aunque «el proyecto de ley no prosperó, [...] fue Romanones quien lo hizo suyo con una serie de retoques, lo mantuvo y lo sacó a la luz» (Ávila, 2011: 441).

En 1901 se incorporan las áreas de geografía e historia, ciencias naturales y dibujo. Numerosas escuelas no implantaron las nuevas materias y pasaron a designarse escuelas incompletas (Ossenbach, 1991). Para la Instrucción Primaria «supuso, además una completa reorganización curricular, un aumento importante de la escolarización obligatoria (antes, de 6 a 9 años; ahora, de 6 a 12)» (Molero, 2000: 63). Erróneamente se asocia la ampliación del rango de edad de seis a doce años para el periodo obligatorio de escolaridad como un logro de la primera ley educativa franquista, pero «con bastante anterioridad a la Ley del 45 se estableció la ampliación de la escuela obligatoria hasta los 12 años (en 1909)» (Egido, 1995: 79).

\section{LA INSTITUCIÓN LIBRE DE ENSEÑANZA Y LAS MISIONES PEDAGÓGICAS: UNA LABOR EN FAVOR DE LA ENSEÑANZA MUSICAL}

La inclusión de la Música en los programas de estudios oficiales de Instrucción Primaria no hubiese sido posible sin la labor privada llevada a cabo por la Institución Libre de Enseñanza. Con su labor educativa, promovió los beneficios de la enseñanza musical en su intento por desarrollar una formación integral, 
entendida como «una formación de hombres y mujeres en el sentido más amplio y alejado del concepto de instrucción, donde la educación integral atiende a todas las dimensiones humanas (corporal, intelectual, moral y estética)» (Payá, 2007: 19).

La ILE es la primera institución educativa española que entre sus enseñanzas imparte contenidos relacionados con la formación estética, entre los que figuran algunos propios de la Educación Musical. Las actividades realizadas eran sobre todo de canto: «Se cantan canciones populares extranjeras como la Berceuse francesa, y otras cuya letra bien pudiera corresponder a Giner de los Ríos y a D. Gabriel Rodríguez» (Garrido y Pinto, 1996: 161). Junto a esta propuesta, también se incluyen canciones folklóricas propias de los distintos territorios españoles.

Giner de los Ríos y posteriormente Manuel Bartolomé Cossío mostraron una postura de gran aprobación hacia la educación cultural en todas sus facetas y la necesidad de ser desarrollada en los procesos de enseñanza del alumnado. Gracias a ellos, la Educación Musical aparece en el Proyecto de Bases de la Enseñanza Primaria y Segunda Enseñanza de 1932, en el que se alude a la música en Primaria como «una actividad higiénica y educativa y aparece en la Segunda Enseñanza como una enseñanza complementaria junto a la taquigrafía y mecanografía» (Martos, 2013: 46).

Por otra parte, el Patronato de las Misiones Pedagógicas creadas en 1931 y presididas por Manuel Bartolomé Cossío tuvo como objetivo «difundir la cultura general, la moderna orientación docente y la educación ciudadana en las aldeas, villas y lugares, con especial atención a los intereses espirituales de la población rural» (Decreto de 29 de mayo, 1931, p. 1034). Dicho de otro modo, la labor de las Misiones Pedagógicas pretendía cubrir las lagunas culturales de los habitantes de las zonas rurales que debido a su localización no podían beneficiarse de la aportación de la cultura (Sánchez, 2013). Pero dicha finalidad no consistió en un proceso para alfabetizar o culturizar sin más a la población rural, sino que «pretendía despertar el interés de la población por la lectura y la cultura y crear así un hábito que se mantuviese firme al término de las misiones» (Gimeno, 2011: 166). Por ello, las actividades culturales y musicales tomaban como punto de partida los gustos de los habitantes para poco a poco ir ampliando el abanico de posibilidades y para ello se crearon distintos servicios. En relación con la música destacar el coro-teatro y las audiciones de obras tanto populares como cultas (Canes, 1993). Así pues, entre el repertorio se incluían tanto canciones folklóricas como canto gregoriano u obras de compositores como Mozart, Schubert o Debussy (Guerra, 2008).

\section{LA LEY DE 1945 Y LOS CUESTIONARIOS NACIONALES DE 1953: UNA EDUCACIÓN DIFERENCIADA POR RAZÓN DE GÉNERO}

En 1939, el nuevo Gobierno nacional-católico emprendió una reforma del sistema educativo. No fue hasta seis años después cuando se promulga la ley de 
17 de julio de 1945 basada en tres pilares que respondían a los ideales de la nueva sociedad: nación, catolicismo y familia. En el propio documento se justifican los motivos de la necesidad de un cambio total en la educación, al mismo tiempo que se acomete duramente contra los ideales de la Ilustración y el Racionalismo, así como con la labor escolar realizada durante la II República. Así pues, la norma (Ley de 17 de julio, 1945) afirma que:

La etapa republicana de mil novecientos treinta y uno llevó a la Escuela a una radical subversión de valores. La legislación de este periodo puso su mayor empeño en arrancar de cuajo el sentido cristiano de la educación, y la Escuela sufrió una etapa de influencias materialistas y desnacionalizantes que la convirtieron en un campo de experimentación para la más torpe política, negadora del ser íntimo de nuestra conciencia histórica (pp. 385-386).

La Enseñanza Primaria se reestructura en cuatro periodos de acuerdo a la edad del alumnado: maternal/parvularios (0-6 años), elemental (6-10 años), perfeccionamiento (10-12 años) e iniciación profesional (12-14 años). Solo el elemental y el de perfeccionamiento eran de carácter obligatorio y, por tanto, los otros dos restantes voluntarios. Al amparo de este marco legislativo, el 6 de febrero de 1953 se publica la Orden Ministerial que establece los Cuestionarios Nacionales para la Enseñanza Primaria cuya finalidad es la de regir todas «las actividades didácticas de las escuelas primarias, siendo obligatorios para las oficiales y orientadores para las privadas» (Cuestionarios Nacionales, 1953, p. 5).

Los propios Cuestionarios Nacionales hicieron explícita la voluntad del Gobierno de acabar con la coeducación de sexos en las aulas, al apostar «contra un igualitarismo didáctico, y en general, educativo, que pugna con la Naturaleza» (Cuestionarios Nacionales, 1953, pp. 14-15). Excepcionalmente, se podían configurar escuelas mixtas cuando el número de alumnado impidiera la creación de aulas separadas o cuando por razones de desplazamiento fuera imposible acudir a una escuela de acuerdo al género correspondiente.

Esta concepción diferenciada de la educación también afectó a las áreas disciplinares desarrolladas en los Cuestionarios (Tabla 1). Así pues, se establecieron áreas comunes, áreas diferenciadas y, por último, la misma área, pero con distintos contenidos dependiendo de si se iba impartir en una escuela de niños o de niñas (López, Vicente y Barea, 2018). 
ALBA MARÍA LÓPEZ MELGAREJO

EL PROGRAMA DE MÚSICA DE LAS ESCUELAS DE NIÑAS DURANTE LA DICTADURA FRANQUISTA: UNA MIRADA A LOS CUESTIONARIOS NACIONALES DE 1953

\begin{tabular}{|c|c|}
\hline Áreas comunes & Áreas masculinas \\
\hline $\begin{array}{c}\text { Lengua Española } \\
\text { Matemáticas } \\
\text { Formación del Espíritu Nacional } \\
\text { Geografía } \\
\text { Historia de España } \\
\text { Ciencias de la Naturaleza } \\
\text { Derecho } \\
\text { Dibujo }\end{array}$ & $\begin{array}{l}\text { Formación política } \\
\text { Educación Física } \\
\text { Canto } \\
\text { Trabajos manuales }\end{array}$ \\
\hline
\end{tabular}

\subsection{La música para las niñas en los Cuestionarios Nacionales}

La propuesta de contenidos musicales realizada por el Gobierno nacional en 1953 y recogida en los Cuestionarios Nacionales muestra diferencias significativas de acuerdo al sexo del alumnado, es decir, para niños y para niñas. Los contenidos musicales se desarrollaron para el alumnado masculino bajo el título de Cuestionario de Canto. En cambio, la nominación asignada para las niñas fue Programa de Música para las Escuelas de niñas.

Los contenidos a trabajar, así como la metodología, diferían mucho uno de otro, al igual que el desarrollo del texto contenido en cada uno de los Cuestionarios. Ambos programas aparecen divididos en tres periodos, pero la denominación de los mismos es diferente para uno y otro sexo. El programa para las niñas marcaba una etapa de enseñanza elemental, un segundo periodo de perfeccionamiento y un último de iniciación profesional. Mientras que la estructura para el programa de canto masculino aparece dividida en función de la edad: niños de seis a ocho años, de nueve a once y de doce a catorce.

El programa femenino se centra en la formación coral a lo largo de los tres periodos educativos. Incluso se puede afirmar de manera fehaciente que se propone una formación basada en clichés propios de lo que debía ser una buena mujer en la época. Por ello, el canto se orienta a realizar dicha actividad lo más naturalmente posible, sin gritar, desarrollando el gusto por lo que en la época se consideraba la buena canción (Cuestionarios Nacionales, 1953).

En relación con los contenidos musicales para las niñas, explícitamente, solo se propone trabajar el canto. El movimiento, la instrumentación o el lenguaje musical no tuvieron cabida en este plan de estudios. Este dato coincide con la realidad ofrecida para los niños para quienes tampoco se desarrollaron actividades de movimiento e instrumentación, aunque el lenguaje musical sí que adquirió una 
presencia significativa en los contenidos destinados a los niños y el movimiento estuvo presente en el área de Educación Física, pero dotado de una orientación fuertemente deportiva. Si bien, en el Programa de Música de las niñas se realiza una mínima reseña al lenguaje musical en el periodo de iniciación profesional, aunque es sorprendente el nivel musical tan bajo que representa esta propuesta de acuerdo a la edad de las alumnas a la que se estaba dirigiendo. Así pues, el documento señala que para las niñas de entre doce y catorce años que estaban cursando este periodo «si es posible, en este grado se iniciará ligeros y elementales conocimientos de solfeo, clave de sol, las notas, las figuras y compases elementales» (Cuestionarios Nacionales, 1953, p. 96).

Aunque se ha aludido que no se proponen contenidos propios de movimiento, entre las orientaciones metodológicas que se establecen para la etapa elemental para la formación musical de las niñas se indica que las canciones deben ser animadas o ir acompañadas de movimiento junto a canciones de texto sencillo y fácil entonación. Entre estas orientaciones se alude a la necesidad de que la maestra debe ofrecer un buen ejemplo vocal y no puede entonar de manera incorrecta. Así mismo, se debe enseñar la canción a modo de eco, es decir, la maestra canta una frase y las alumnas la repiten con una «afinación exacta» (Cuestionarios Nacionales, 1953, p. 92).

A diferencia de lo que ocurre en los contenidos españoles actuales, en este momento se determinan las canciones que forman parte de las enseñanzas mínimas. Para este periodo se enumeran once que responden a distintas categorías. Existe un predominio por la inclusión de canciones de corro, lo que se justifica a partir de la orientación metodológica incluida a su vez que aludía a la necesidad de acompañar las canciones con movimiento. Entre las canciones figuran: «Que Llueva, que Llueva»; «El Patio de mi Casa»; «Baile de la Carrasquilla»; «Dice mi Tía doña Inés» $\mathrm{y}$ «Este Corro es un Jardín». También están presentes canciones donde prima el componente religioso ( $«$ Oh! Virgen Purísima» o «El Romance de los Pajaritos de San Antonio de Padua») y un villancico («Chin, chin, chiquirritín»). «Cucú Cantaba la Rana» y el «Balcón de Palacio» son las dos únicas propuestas en compás de tres por cuatro. Por último, aludir a la canción narrada «Margarita Va por Agua» y «Quieren Ver como el Chacarero».

El periodo de perfeccionamiento supone, como la propia nominación de la etapa indica, una mejora en el proceso de canto a partir de los conocimientos adquiridos durante el periodo elemental. Durante la interpretación de la canción se comienza a dar importancia a aspectos musicales como realizar el apoyo en el acento del compás, optando por la repetición de un modo continuo, como el método idóneo para que las niñas aprendan a interpretar adecuadamente las distintas canciones. Si bien, tal como indica el propio documento, se pretende «ir haciéndoles comprender lo que 
es «buena canción», para ir educándoles el gusto, y, sobre todo, no se permitirá que canten gritando ni con estridencias» (p. 95).

Entre las doce canciones que se incluyen en el programa de canto de niñas para esta etapa figuran religiosas («Ave María»), romances («El Enrame de la Fuente»), canciones de cuna («Calla, Niña, Calla»), Himnos propios de la Falange («En Pie, Flechas de España»), villancicos para la etapa de Navidad («Campanas de Belén»), humorísticas («Fray Antón Tenía una Burra») y otras propias de la tradición oral como «Mambrú se Fue la guerra», «Tiendo mi Pañuelo», «El Ramito Verde», «Cómo Quieres que Tenga la Cara blanca», «Rosalinda» o "Carta del Rey».

El programa de estudios para este periodo es menos preciso que en las dos etapas anteriores probablemente a consecuencia de que esta etapa contaba con un carácter optativo o voluntario frente a las dos anteriores que eran obligatorias. Las orientaciones metodológicas para enseñar a cantar son las mismas que en el anterior y así lo hacen explícito los propios Cuestionarios Nacionales. Como nueva inclusión hay que destacar que se propone llevar a cabo una iniciación a los conceptos más básicos del lenguaje musical si se considera que es posible. Entre los contenidos a trabajar se indica que se ha de dar a conocer a las féminas el «pentagrama, clave de sol, las notas, las figuras y compases elementales» (p. 96). Se ha de recordar que este periodo era cursado por niñas con edades comprendidas entre doce y catorce años. Por ello, es destacable el nivel tan elemental y básico de las enseñanzas musicales propuestas que hoy coincidirían con las que se imparten en el primer nivel de la Educación Primaria. También, en relación con el lenguaje musical, se ha de mencionar que en el caso del plan de estudios para los niños, incluso para el periodo elemental destinado a los menores entre 6 y 8 años, eran de una dificultad mucho mayor en comparación con la propuesta de contenidos para el periodo de iniciación profesional de las niñas, al incluir para estos actividades de discriminación auditiva, conocimiento de los parámetros de sonido (intensidad y altura), ejercicios rítmicos o marcar compases de dos tiempos mientras se entona una canción.

Las canciones son muy variadas en cuanto a su tipología: romances («El Conde Olinos»); canciones de trabajo ( «Canción de Siega de Salamanca» o «Canción de recogida de la aceituna de Ciudad Real»); humorísticas («Don Melitón»); villancicos («Estando la Virgen María»); himnos («En Pie, Camaradas»); canciones de cuna (propias de Palencia); religiosas, incluyendo el canto gregoriano dentro de estas ( Flos Virginum»), y canciones procedentes de distintas regiones, como el «Villancico del Vestido» (Andalucía), «El Picotín» (Burgos), «María de las Nieves» (Granada) o «Como Vives tan alta» (Toledo), son las propuestas por el Gobierno nacional que las niñas debían aprender en la escuela durante el periodo de iniciación profesional. 
ALBA MARÍA LÓPEZ MELGAREJO

EL PROGRAMA DE MÚSICA DE LAS ESCUELAS DE NIÑAS DURANTE LA DICTADURA FRANQUISTA:

UNA MIRADA A LOS CUESTIONARIOS NACIONALES DE 1953

\section{CONSIDERACIONES FINALES}

A lo largo de las tres etapas en las que se dividía la enseñanza musical para las niñas en los Cuestionarios Nacionales de 1953, podemos encontrar una línea bien definida de la intención educativa para cada periodo. En el primero, elemental, se centra en el aprendizaje de las canciones a través de ejercicios de eco; en el segundo periodo, de perfeccionamiento, se destaca la manera idónea según los criterios estéticos del régimen para una correcta interpretación de las canciones; por último, en el tercer periodo, denominado de Iniciación Profesional, se introduce a las alumnas en conceptos básicos del Lenguaje Musical que en la actualidad son enseñados en el primer año de Educación Primaria.

Respecto a las diferencias en el programa dirigido a niños se aprecian tres diferencias significativas: contenidos con diferente terminología, metodologías diferentes según el sexo y mayor número de contenidos del lenguaje musical y de mayor dificultad para niños que para niñas. En este sentido, coincidimos con Martín (2001) que señala una diferencia significativa en la propuesta de niños con relación a las niñas, en tanto en cuanto, estos tenían una proporción mayor de temas propios del nacionalcatolicismo.

La propuesta de contenidos para las niñas está marcada por un predominio total del canto obviando aspectos musicales como la escucha, la instrumentación o el movimiento. Si bien, este último estaría presente en aquellas canciones que lo requerían como fueron las canciones de corro. A pesar de ello, el repertorio de las niñas era muy variado con canciones agrupadas en diversas categorías como los romances, religiosas, himnos, villancicos o humorísticas.

\section{BIBLIOGRAFÍA}

Araque, N. (2009). La educación en la Constitución de 1812: Antecedentes y consecuencias. Revista de la Facultad de Ciencias Sociales y Jurídicas de Elche, 1(1), 1-21.

Ávila, A. (1990). La enseñanza primaria a través de los planes y programas escolares en la legislación española durante el siglo XIX. Cuestiones Pedagógicas. Revista de Ciencias de la Educación, 6-7, 215-230.

Canes, F. (1993). Las misiones pedagógicas: educación y tiempo libre en la Segunda República. Revista Complutense de Educación, 4(1), 147-168.

Constitución Política de la Monarquía Española (1812). Recuperado de http://www. congreso.es/constitucion/ficheros/historicas/cons_1812.pdf

Cuestionarios Nacionales para la Enseñanza Primaria (1953). Madrid: Servicio de Publicaciones del Ministerio de Educación Nacional.

Domínguez, M. (1990). La educación durante la Ilustración Española. Norba. Revista de Historia, 10, 173-186. 
Egido, I. (1995). La evolución de la enseñanza primaria en España: Organización de la etapa y programa de estudios. Tendencias Pedagógicas, 1, 75-86.

Garrido, J. Á. y Pinto, A. (1996). La educación estética en la Institución Libre de Enseñanza. Revista Interuniversitaria de Formación del Profesorado, 27, 151-166.

Gimeno, J. (2011). Esbozo de una utopía: las Misiones Pedagógicas de la II República española (1931-1939).F@ro: Revista Teórica del Departamento de Ciencias de la Comunicación, 13, 160-177.

Guerra, A. (2008). Las Misiones Pedagógicas y la Barraca: la cultura en la II República. Letra Internacional, 100, 5-12.

Lázaro, L. (1993). La Revolución Francesa y su influencia en la educación española. Revista de Educación, 300, 399-420.

Ley de 17 de julio de 1945 sobre Educación Primaria. Boletín Oficial del Estado, n. ${ }^{\circ}$ 199, 18 de julio de 1945, 385-416.

López, A. M.; Vicente, G. y Barea, E. M. (2018). La danza en la Educación Primaria del Franquismo: una visión documental a partir de los Cuestionarios Nacionales. En A. M. Díaz, Á. Calvo y C. Pedrero (Coords.), Danza, investigación, educación (pp. 29-40). Granada: Libargo.

Martín, M. J. (2001). Las canciones infantiles de transmisión oral en Murcia durante el siglo $X X$ (tesis doctoral). Universidad de Murcia, España.

Martos, E. (2013). La normativa legal sobre educación musical en la España Contemporánea. Espiral. Cuadernos del Profesorado, 6(12), 43-50.

Molero, A. (2000). La formación del maestro español, un debate histórico permanente. Revista de Educación, n. ${ }^{\circ}$ extraordinario, 59-82.

Morales, Á. (2008). La Educación Musical en Primaria durante la LOGSE en la Comunidad de Madrid: Análisis y Evaluación (tesis doctoral). Universidad Autónoma de Madrid, Madrid.

Ossenbach, G. (1991). Resistencia a las innovaciones curriculares en la primera mitad del siglo XX en España. Revista de Educación, 293, 383-388.

Payá, A. (2007). La actividad lúdica en la Historia de la Educación Española Contemporánea (tesis doctoral). Universitat de Valencia, Valencia.

Puelles, M. (1999). Educación e ideología en la España contemporánea. Madrid: Tecnos.

Sánchez, C. (2013). Las Misiones pedagógicas durante la II República Española: la transmisión de los legados culturales en los territorios fronterizos de la Pedagogía Social. RES Revista de Educación Social, 17, 1-7. 
